\title{
Phonon spectrum and the maintenance condition of crystalline beams
}

\author{
Xiao-Ping Li \\ Skyworks Solutions, Inc., 2427 West Hillcrest Drive, Newbury Park, California 91320, USA \\ Hayato Enokizono and Hiromi Okamoto \\ Department of Quantum Matter, Graduate School of Advanced Sciences of Matter, Hiroshima University, \\ 1-3-1 Kagamiyama, Higashi-Hiroshima 739-8530, Japan \\ Yosuke Yuri \\ Takasaki Advanced Radiation Research Institute, Japan Atomic Energy Agency, \\ 1233 Watanuki-machi, Takasaki 370-1292, Japan \\ Andrew M. Sessler \\ Lawrence Berkeley National Laboratory, Berkeley, California 94720, USA \\ Jie Wei* \\ Brookhaven National Laboratory, Upton, New York 11973, USA
}

(Received 18 July 2005; published 9 March 2006)

\begin{abstract}
It has been shown that the maintenance condition for a crystalline beam requires that there be no resonance between the crystal's phonon frequencies, and the frequency associated with a beam moving through a lattice of $N_{\text {sp }}$ periods. This resonance can be avoided provided that the phonon frequencies all are below half the lattice frequency. Here, we study in detail the phonon modes of several crystalline beams. The analytic results obtained in the smooth approximation are compared with numerical evaluations employing Fourier transform of the molecular dynamics (MD) modes. The stability of various crystalline structures is examined through systematic MD simulations based on several different lattice designs. The maintenance condition, when combined with either the simple analytic theory or the numerical evaluation of phonon modes, exhibits excellent agreement with the MD calculations of crystal stability. A confirmed maintenance condition, derived from linear-resonance criteria, is that the lattice frequency must not equal the sum of any two phonon frequencies.
\end{abstract}

DOI: 10.1103/PhysRevSTAB.9.034201

PACS numbers: 52.59.Sa, 29.20.Dh, 61.50.-f, 63.20.Ls

\section{INTRODUCTION}

In the work that first considered the properties of crystalline beams ("crystals") in "real" circular accelerators or storage rings, i.e., circular machines that allow the formation and maintenance of the crystals, we proposed two criteria. First, the machine cannot be a weak-focusing one (i.e., a constant gradient at all azimuths), but must be a strong-focusing one operating below the transition energy [1]. The second, a maintenance condition for a crystalline beam, requires that there is not a linear resonance between the crystal's phonon frequencies and the frequency associated with a beam moving through a lattice of $N_{\text {sp }}$ periods $[2,3]$. This paper is devoted to studying this second criterion, the maintenance condition.

Previously, the maintenance condition stated that the lattice's superperiodicity must be larger than $2 \sqrt{2}$ times the maximum "bare" transverse (betatron) tune. For a typical high-density crystalline beam (i.e., threedimensional crystal of multiple layers), the maximum phonon frequency is nearly $\sqrt{2}$ times the higher single-

*Electronic address: jwei@bnl.gov particle betatron tune. Resonance can be avoided provided that the phonon frequencies all are below half the lattice frequency and, generally, we find that this is so. In this paper, we closely examine cases from low- to high-beam density, and study in detail phonon modes and the maintenance condition.

As is well known, a variety of Coulomb crystals have already been realized in compact ion traps by applying the laser cooling method [4-6]. On the other hand, extensive theoretical efforts for the last decade have shown that beam crystallization in a circular accelerator is much more complicated due mainly to the existence of dipole fields that yield strong momentum dispersion $[3,7,8]$. In a dispersive environment, we always need a special cooling force to stabilize multidimensional crystalline structures. The importance of momentum dispersion in the ultracold regime has been experimentally confirmed with a circular Paul trap where Coulomb crystals can be maintained only at very low kinetic energy (below a few eV) [9]. Since any beams in ring accelerators are exposed to much stronger momentum dispersion and complex external fields, we must overcome severe obstacles toward beam crystallization in practice. In the present simulation study, an ideal 3D 
cooling force is assumed to reach ordered states because the stability of crystalline beams is of primary interest here. We also pay little attention to the cooling process that brings an ordinary "hot" beam to an ordered state; in other words, we do not consider what happens before a crystalline ground state is reached.

The phonon modes are analyzed under the so-called smooth approximation for a one-dimensional (1D) structure and numerically in general for multidimensional ground-state structures. We then undertake a computational evaluation employing Fourier transform of particle trajectory simulated with the molecular dynamics (MD) method. We show that the maintenance condition, based on the evaluated phonon modes, is in excellent agreement with the MD simulations on stability; that is, it can explain those cases we discovered that violate the simple criterion of having all phonon modes below half the lattice frequency.

The paper is organized as follows: In Sec. II, we present the Hamiltonian employed, give various parameters, and analytically evaluate the phonon spectrum. In Sec. III, we introduce MD and employ it to determine, by Fourier transform, the phonon spectrum. We confront in MD the spectra with 1D crystals that are stable, or unstable. We further investigate, in Sec. IV, the stability of crystalline beams with multiple dimensions, adopting six different lattice structures. Simulation results on the two existing storage rings, TSR and ASTRID, are also discussed. Finally, Sec. V has the discussions and a brief summary.

\section{ANALYTICAL PHONON SPECTRUM}

To calculate the approximate phonon modes of a crystalline beam, we assume that the external bending and focusing forces are uniform in time (the smooth approximation). The rest-frame motions of particles interacting through the Coulomb fields then are governed by the Hamiltonian [10]

$$
\begin{aligned}
H= & \frac{1}{2} \sum_{\ell}\left(P_{x, \ell}^{2}+P_{y, \ell}^{2}+P_{z, \ell}^{2}\right)-\sum_{\ell} \gamma x_{\ell} P_{z, \ell} \\
& +\frac{1}{2} \sum_{\ell}\left(\nu_{x}^{2} x_{\ell}^{2}+\nu_{y}^{2} y_{\ell}^{2}\right)+V_{C},
\end{aligned}
$$

where $\nu_{x}$ and $\nu_{y}$ are the transverse betatron tunes, and $\gamma$ is the Lorentz factor. In Eq. (1), all canonical variables are scaled as dimensionless by expressing the time, $t$, in units of $R / \beta \gamma c$, the spatial coordinates $x, y$, and $z$ in units of the characteristic interparticle distance $\xi \equiv\left(r_{0} R^{2} / \beta^{2} \gamma^{2}\right)^{1 / 3}$, and the energy in units of $\beta^{2} \gamma^{2} e^{2} / \xi$, where $\beta c$ is the velocity of the reference particle, $r_{0} \equiv e^{2} / m_{0} c^{2}$ is its classical radius, and $R$ is the bending radius of the ring under the dipole magnetic field [1]. The Coulomb potential is given by

$$
V_{C}=\frac{1}{2} \sum_{\ell \neq m} \frac{1}{\left|\mathbf{r}_{\ell}-\mathbf{r}_{m}\right|}
$$

where

$$
\left|\mathbf{r}_{\ell}-\mathbf{r}_{m}\right|=\left[\left(x_{\ell}-x_{m}\right)^{2}+\left(y_{\ell}-y_{m}\right)^{2}+\left(z_{\ell}-z_{m}\right)^{2}\right]^{1 / 2} .
$$

The only nonlinear terms in the canonical equations of motion are derivatives of $V_{C}$ that can be Taylor expanded around the equilibrium positions, retaining only the linear terms. Writing the spatial coordinates of $\ell$ th ion in a crystalline state as $\left(X_{\ell}, Y_{\ell}, Z_{\ell}\right)$, we have

$$
\begin{array}{rlrl}
x_{\ell} & =X_{\ell}+\delta x_{\ell}, & \delta x_{\ell}=\tilde{x}_{\ell} \exp \left[i\left(\omega t-k Z_{\ell}\right)\right], \\
y_{\ell}=Y_{\ell}+\delta y_{\ell}, & \delta y_{\ell}=\tilde{y}_{\ell} \exp \left[i\left(\omega t-k Z_{\ell}\right)\right], \\
z_{\ell}=Z_{\ell}+\delta z_{\ell}, & \delta z_{\ell}=\tilde{z}_{\ell} \exp \left[i\left(\omega t-k Z_{\ell}\right)\right] .
\end{array}
$$

When there are $N$ particles per unit cell of length $L$, we obtain the linearized equations of motion in a storage ring,

$$
\begin{aligned}
\omega^{2} \tilde{x}_{\ell}= & -i \gamma \omega \tilde{z}_{\ell}+\left(\nu_{x}^{2}-\gamma^{2}\right) \tilde{x}_{\ell}+\sum_{n=-\infty}^{\infty} \sum_{m=1}^{N}\left\{\left[\frac{1}{R_{\ell m n}^{3}}-\frac{3\left(X_{\ell}-X_{m}\right)^{2}}{R_{\ell m n}^{5}}\right]\left[e^{i k\left(Z_{\ell}-Z_{m}-n L\right)} \tilde{x}_{m}-\tilde{x}_{\ell}\right]\right. \\
& \left.-\frac{3\left(X_{\ell}-X_{m}\right)\left(Y_{\ell}-Y_{m}\right)}{R_{\ell m n}^{5}}\left[e^{i k\left(Z_{\ell}-Z_{m}-n L\right)} \tilde{y}_{m}-\tilde{y}_{\ell}\right]-\frac{3\left(X_{\ell}-X_{m}\right)\left(Z_{\ell}-Z_{m}-n L\right)}{R_{\ell m n}^{5}}\left[e^{i k\left(Z_{\ell}-Z_{m}-n L\right)} \tilde{z}_{m}-\tilde{z}_{\ell}\right]\right\}
\end{aligned}
$$

$$
\begin{aligned}
\omega^{2} \tilde{y}_{\ell}= & \nu_{y}^{2} \tilde{y}_{\ell}+\sum_{n=-\infty}^{\infty} \sum_{m=1}^{N}\left\{-\frac{3\left(X_{\ell}-X_{m}\right)\left(Y_{\ell}-Y_{m}\right)}{R_{\ell m n}^{5}}\left[e^{i k\left(Z_{\ell}-Z_{m}-n L\right)} \tilde{x}_{m}-\tilde{x}_{\ell}\right]\right. \\
& \left.+\left[\frac{1}{R_{\ell m n}^{3}}-\frac{3\left(Y_{\ell}-Y_{m}\right)^{2}}{R_{\ell m n}^{5}}\right]\left[e^{i k\left(Z_{\ell}-Z_{m}-n L\right)} \tilde{y}_{m}-\tilde{y}_{\ell}\right]-\frac{3\left(Y_{\ell}-Y_{m}\right)\left(Z_{\ell}-Z_{m}-n L\right)}{R_{\ell m n}^{5}}\left[e^{i k\left(Z_{\ell}-Z_{m}-n L\right)} \tilde{z}_{m}-\tilde{z}_{\ell}\right]\right\},
\end{aligned}
$$




$$
\begin{aligned}
\omega^{2} \tilde{z}_{\ell}= & i \gamma \omega \tilde{x}_{\ell}+\sum_{n=-\infty}^{\infty} \sum_{m=1}^{N}\left\{-\frac{3\left(X_{\ell}-X_{m}\right)\left(Z_{\ell}-Z_{m}-n L\right)}{R_{\ell m n}^{5}}\left[e^{i k\left(Z_{\ell}-Z_{m}-n L\right)} \tilde{x}_{m}-\tilde{x}_{\ell}\right]-\frac{3\left(Y_{\ell}-Y_{m}\right)\left(Z_{\ell}-Z_{m}-n L\right)}{R_{\ell m n}^{5}}\right. \\
& \left.\times\left[e^{i k\left(Z_{\ell}-Z_{m}-n L\right)} \tilde{y}_{m}-\tilde{y}_{\ell}\right]+\left[\frac{1}{R_{\ell m n}^{3}}-\frac{3\left(Z_{\ell}-Z_{m}-n L\right)^{2}}{R_{\ell m n}^{5}}\right]\left[e^{i k\left(Z_{\ell}-Z_{m}-n L\right)} \tilde{z}_{m}-\tilde{z}_{\ell}\right]\right\}
\end{aligned}
$$

where $R_{\ell m n}=\sqrt{\left(X_{\ell}-X_{m}\right)^{2}+\left(Y_{\ell}-Y_{m}\right)^{2}+\left(Z_{\ell}-Z_{m}-n L\right)^{2}}$, $\ell=1, \ldots, N$, and $R_{\ell m n}=0$ term is excluded from the double sum.

\section{A. 1D crystalline beam}

The configuration of a Coulomb crystal is $1 \mathrm{D}$ at a low line density [1]. Then, we insert $N=1$ and $(X, Y, Z)=$ $(0,0,0)$ in Eqs. (5)-(7). The phonon bands can readily be calculated as

$$
\begin{aligned}
& \omega_{1}^{2}=\frac{1}{2}\left\{\nu_{x}^{2}+\Omega^{2}+\sqrt{\left(\nu_{x}^{2}+\Omega^{2}\right)^{2}-8 \Omega^{2}\left(\nu_{x}^{2}-\gamma^{2}-\Omega^{2}\right)}\right\}, \\
& \omega_{2}^{2}=\nu_{y}^{2}-\Omega^{2}, \\
& \omega_{3}^{2}=\frac{1}{2}\left\{\nu_{x}^{2}+\Omega^{2}-\sqrt{\left(\nu_{x}^{2}+\Omega^{2}\right)^{2}-8 \Omega^{2}\left(\nu_{x}^{2}-\gamma^{2}-\Omega^{2}\right)}\right\},
\end{aligned}
$$

where

$$
\Omega^{2}=2 \sum_{n=1}^{\infty} \frac{1-\cos (k n / \Lambda)}{(n / \Lambda)^{3}} \geq 0
$$

with $\Lambda$ being the scaled dimensionless line density defined by $\Lambda=N / L$, and $k$ varies from $-\pi \Lambda$ to $\pi \Lambda$. The actual line density $\lambda$ in the laboratory frame can be related to $\Lambda$ as $\lambda=\Lambda /(\gamma \xi)$. Since all particles are aligned on axis, $\Lambda^{-1}$ is simply equal to the scaled interparticle distance in the present $1 \mathrm{D}$ case. The phonon bands then satisfy the relation $\sum_{j=1}^{3} \omega_{j}^{2}=\nu_{x}^{2}+\nu_{y}^{2}, \omega_{1}^{2}+\omega_{3}^{2}=\nu_{x}^{2}+\Omega^{2}$ and $\omega_{3}^{2} \leq \omega_{1}^{2}$.

The frequency $\omega_{2}$ corresponds to the motion polarized in

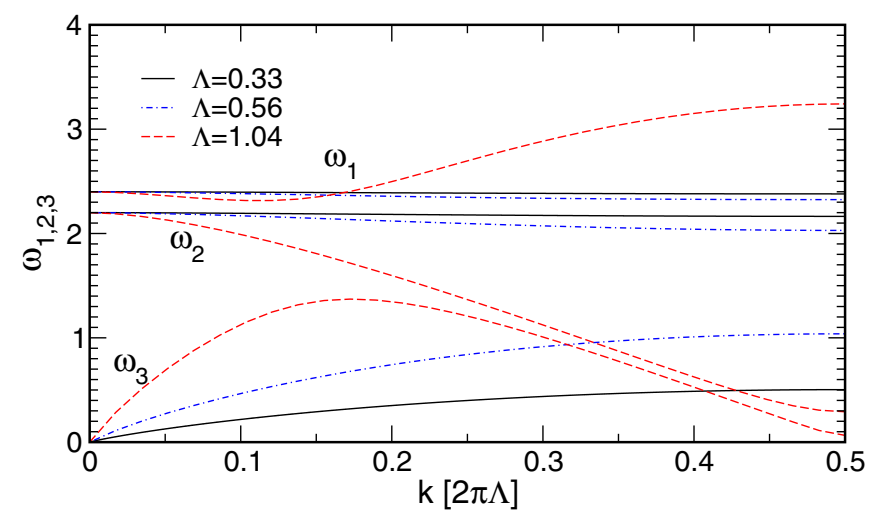

FIG. 1. (Color) Dispersion function evaluated under the smooth approximation of three $1 \mathrm{D}$ crystalline beams with $\Lambda=0.33$, 0.56 , and 1.04. The horizontal and vertical tunes are $\nu_{x}=2.4$ and $\nu_{y}=2.2$, respectively, and $\gamma=1.000016$. $y$, while the frequencies, $\omega_{1}$ and $\omega_{3}$, correspond to the motion coupled in the $x$ and $z$ directions.

A test particle deviating from its equilibrium position experiences defocusing forces both horizontally $(x)$ and vertically $(y)$, and focusing force longitudinally $(z)$ through Coulomb interaction. At a low beam density $(\Lambda=0.33$ case of Figs. 1 and 2), the phonon frequencies in both modes 1 and 2 shift downwards from the base tune, while the frequency in mode 3 shifts upwards from 0 (Fig. 1). Figure 2 shows the typical density of states.

When the beam's density increases, the extent of frequency downshift in mode 2, and upshift in mode 3, also increases, as illustrated in Figs. 1 and 2. Because of the coupling of horizontal and longitudinal motion, the frequency in mode 1 also can shift upwards from the base tune $\nu_{x}$. The amount of upward frequency shift reaches a maximum when the density approaches the threshold of a string-to-zigzag transition (Fig. 2). The maximum phonon frequency for a stable $1 \mathrm{D}$ crystalline beam satisfies

$$
\omega_{1,3, \max } \leq \sqrt{\nu_{x}^{2}+\nu_{y}^{2}}, \quad \omega_{2, \max } \leq \nu_{y}
$$

The maximum frequency is achieved when $k=\pi \Lambda$.

It is interesting to study the particle motion in real space under different modes of eigenfrequency, especially when the density approaches the value for a structure transition. The linearized equations of motion for a 1D crystal are given by

$$
\left\{\begin{array}{l}
\left(-\omega^{2}+\nu_{x}^{2}-\gamma^{2}-\Omega^{2}\right) \tilde{x}-i \gamma \omega \tilde{z}=0 \\
\left(-\omega^{2}+\nu_{y}^{2}-\Omega^{2}\right) \tilde{y}=0 \\
i \gamma \omega \tilde{x}+\left(-\omega^{2}+2 \Omega^{2}\right) \tilde{z}=0
\end{array}\right.
$$

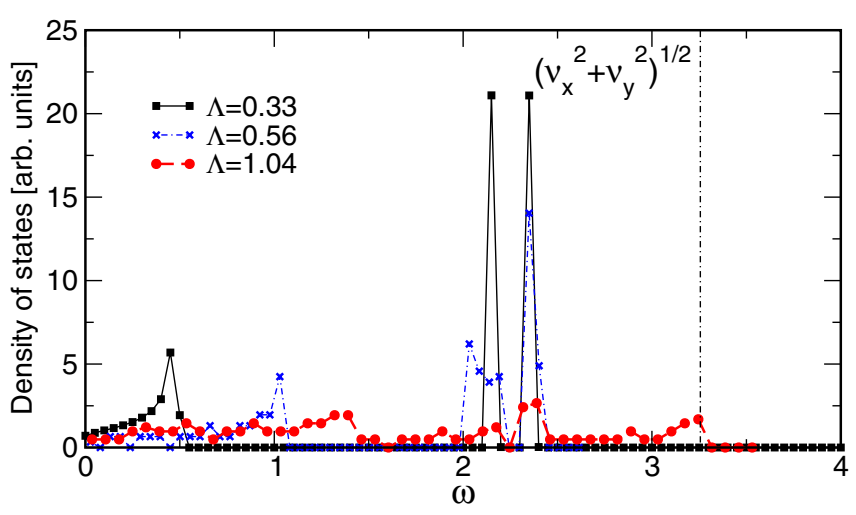

FIG. 2. (Color) Density of states corresponding to Fig. 1 evaluated under the smooth approximation at three beam densities with $\Lambda=0.33,0.56$, and 1.04 . 
The eigenfrequency $\omega_{2}$ corresponds to the motion in the vertical direction $(\tilde{x}=\tilde{z}=0$ and $\tilde{y} \neq 0)$. The eigenfrequencies $\omega_{1}$ and $\omega_{3}$ correspond to the coupled motion $(\tilde{y}=0, \tilde{x} \neq 0$, and $\tilde{z} \neq 0$ ) with the motions in the horizontal and longitudinal directions in general $90^{\circ}$ out-of-phase.

Consider the motion at $k=\pi \Lambda$ that corresponds to the maximum frequency upshift of mode $1\left(\omega_{1}\right)$ and downshift of modes 2 and $3\left(\omega_{2}\right.$ and $\left.\omega_{3}\right)$ (Fig. 1). At this $k$ value, nearest-neighbor particles $\ell$ and $\ell+1$ move in the opposite phase in each direction. In the case that the horizontal focusing is stronger than the vertical focusing, i.e., $\nu_{x}^{2}-$ $\gamma^{2}>\nu_{y}^{2}$, the mode $\omega_{2}$ first approaches instability when the density increases so that $\nu_{y}^{2} \approx \Omega^{2}$ and $\omega_{2} \approx 0$. The threshold density corresponds to [3]

$$
\Lambda_{\mathrm{th}}=\left(\frac{\nu_{y}^{2}}{4.2}\right)^{1 / 3}, \quad \text { for } \nu_{x}^{2}-\gamma^{2}>\nu_{y}^{2}
$$

Below this threshold, nearest-neighbor particles $\ell$ and $\ell+$ 1 move in the opposite direction in $y$. Above this threshold, the $1 \mathrm{D}$ structure is unstable in $y$; the stable structure is a $2 \mathrm{D}$ zigzag extending in $y$.

Similarly, in the case that the vertical focusing is stronger than the horizontal focusing, i.e., $\nu_{y}^{2}>\nu_{x}^{2}-\gamma^{2}$, the mode $\omega_{3}$ first approaches instability when the density increases so that $\nu_{x}^{2}-\gamma^{2} \approx \Omega^{2}, \omega_{3} \approx 0$, and $\omega_{1} \approx$ $\sqrt{2 \nu_{x}^{2}-\gamma^{2}}$. The threshold density corresponds to

$$
\Lambda_{\mathrm{th}}=\left(\frac{\nu_{x}^{2}-\gamma^{2}}{4.2}\right)^{1 / 3}, \quad \text { for } \nu_{y}^{2}>\nu_{x}^{2}-\gamma^{2}
$$

Below this threshold, nearest-neighbor particles $\ell$ and $\ell+$ 1 move in the opposite direction in $x$ corresponding to $\omega_{3}$ $(\tilde{z}=\tilde{y}=0$ and $\tilde{x} \neq 0)$. Above this threshold, the 1D structure is unstable in $x$; the stable structure is a $2 \mathrm{D}$ zigzag extending in $x$. The mode $\omega_{1}$ corresponds to nearestneighbor particles $\ell$ and $\ell+1$ moving in the opposite direction in $z(\tilde{x}=\tilde{y}=0$ and $\tilde{z} \neq 0)$; it is always stable.

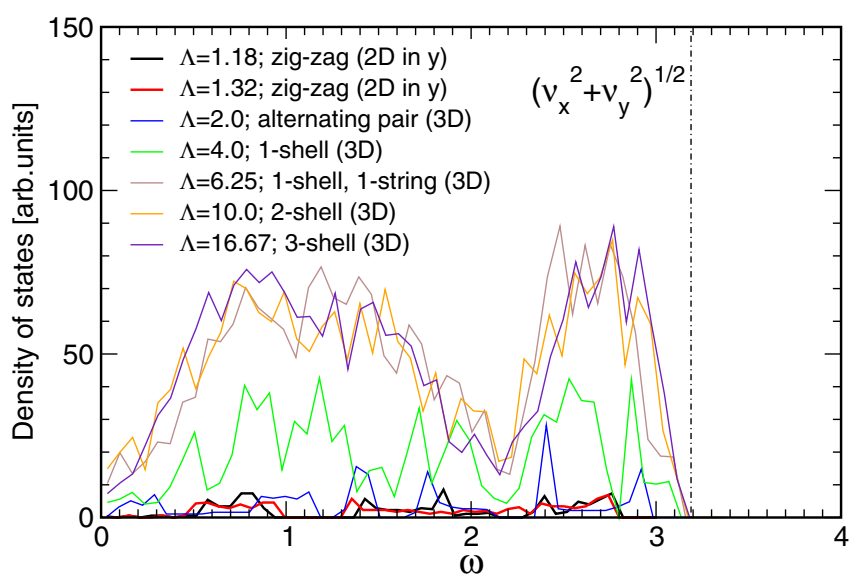

FIG. 4. (Color) Density of states evaluated under the smooth approximation for various $2 \mathrm{D}$ and $3 \mathrm{D}$ crystalline beams. We assume that $\nu_{x}=2.4, \nu_{y}=2.1$, and $\gamma=1.000016$.

\section{B. 2D and 3D crystalline beams}

For a crystalline beam beyond one dimension, the dispersion relation cannot generally be solved analytically. We first need to obtain the equilibrium crystalline structure under the smooth approximation using a MD code. Then, the dispersion relation and the density of states are evaluated with Li et al.'s numerical algorithm [2].

Figure 3 shows the ground-state structure of a threedimensional (3D) crystalline beam. The MD unit cell contains 20 particles, and its length is $L=5$ (thus, $\Lambda=4$ ). At this line density, the ground state is a set of helices formed on a single-shell extending in the $z$ direction.

Figure 4 shows the density of states of various twodimensional (2D) and three-dimensional (3D) crystalline beams. Interestingly, the maximum frequency of the phonon bands always is below the square root of $\nu_{x}^{2}+\nu_{y}^{2}$, i.e.,

$$
\omega_{\max } \leq \sqrt{\nu_{x}^{2}+\nu_{y}^{2}}
$$

This relation is expected to be a general one, independent of the choice of the machine's lattice parameters.

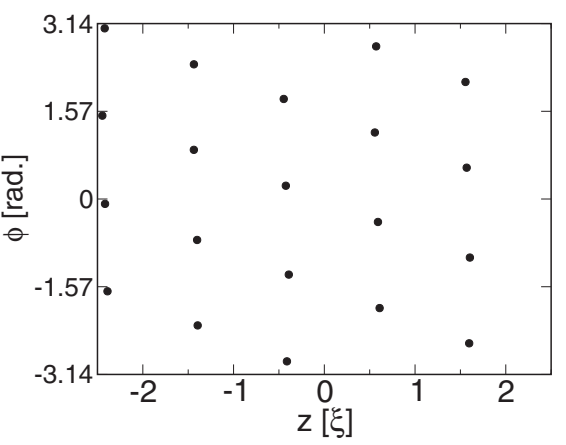

FIG. 3. Projections in the $x-y$ (left) and $z-\phi$ (right) planes of an one-shell, three-dimensional crystalline beam with $\Lambda=4.0$ and 20 particles per unit cell, where $\phi \equiv \tan ^{-1}(y / x)$ is the polar angle. The horizontal and vertical tunes are $\nu_{x}=2.4$ and $\nu_{y}=2.1$, respectively. The beam's Lorentz relativistic factor is $\gamma=1.000016$. 


\section{MAINTENANCE CONDITION OF ONE- DIMENSIONAL CRYSTALS}

The MD method enables us to directly evaluate the phonon spectrum for a real lattice without using the smooth approximation. What we must do is to determine a ground state, and then, evaluate the density of states in the frequency domain by Fourier analyzing the particle trajectory under a small-amplitude vibration. From wide range of choices, we here consider a set of model storage rings, each consisting of 12 FODO cells. To study the latticeperiodicity dependence of the phonon spectrum, we properly specify the field gradients of some quadrupole magnets, controlling the machine's superperiodicity $N_{\mathrm{sp}}$. Six lattices slightly different from each other are taken into account; namely, $N_{\mathrm{sp}}=1,2,3,4,6$, and 12 . For reference, Fig. 5 depicts two of them. We assumed there is uniform bending so that the design beam's orbit is a circle of $1.0 \mathrm{~m}$ in radius.

The maintenance condition based on linear-resonance criteria is that the lattice frequency $\left(N_{\mathrm{sp}}\right)$ must not equal the sum of any two phonon frequencies. In the following subsections, MD results are given of the phonon spectra of $1 \mathrm{D}$ chains circulating in the six model rings. Similarly to the case in Fig. 4, we evaluate the phonon spectrum by Fourier analyzing the small oscillations of particles about the equilibrium orbits. The stability of 1D crystalline beams is determined by tracking the time evolution of the root-mean-squared (rms) emittance. The amount of growth in Fig. 6 represents the ratio of rms emittances before, and 100-turns after, removing the cooling force.
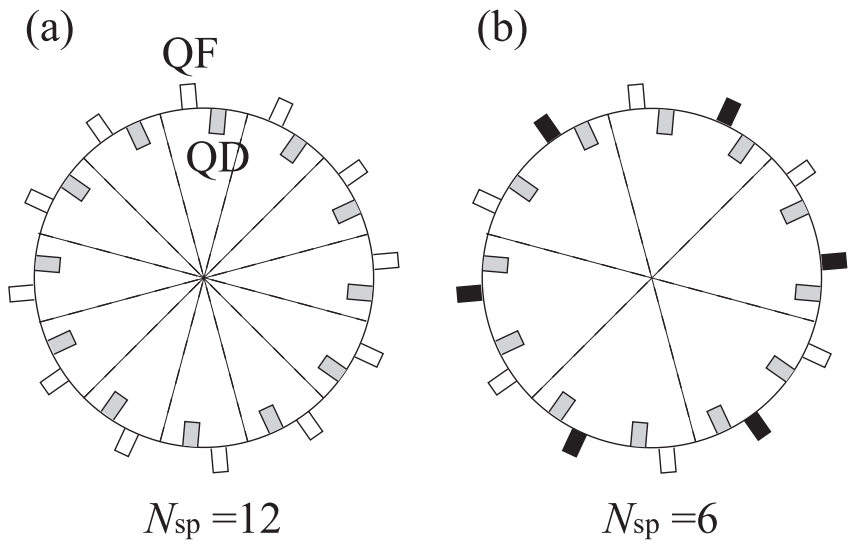

FIG. 5. Schematic layouts of the rings with (a) $N_{\mathrm{sp}}=12$ and (b) $N_{\mathrm{sp}}=6$. These model rings are composed of 12 FODO cells. In case (a), the ring consists of 12 identical FODO cells while, in case (b), focusing-quadrupole (QF) magnets with different field gradients (indicated by white and black squares) were used to reduce the superperiodicity of the ring. A uniform dipole field exists all around the rings, so that the beam's orbit is a circle.
A. $N_{\text {sp }}=12$

The frequency shift of each mode in Fig. 6(a) behaves similarly to that predicted by the smooth approximation theory. As the beam's density increases, the phonon frequency in mode 1 shifts upwards from 2.4, in mode 2 downwards from 2.2, and in mode 3 upwards from 0 . The maximum frequency in mode 1 reaches 2.8 corresponding to the threshold of the 1D-to-2D transition when $\Lambda=0.98$. Since all phonon modes are far from resonance, the 1D crystalline chain always is stable when the density is below this threshold.

$$
\text { B. } N_{\mathrm{sp}}=6
$$

Here, the frequency shifts show the same tendencies as those in which $N_{\mathrm{sp}}=12$. The maximum frequency reaches 2.7 when $\Lambda=0.85$, while the resonance line is located at $\omega_{1}=3$. Thus, the $1 \mathrm{D}$ crystalline chain again is stable when the density is below the 1D-to-2D threshold.

$$
\text { C. } N_{\text {sp }}=4
$$

The phonon frequencies in modes 1 and 3 shift only slightly in the $N_{\mathrm{sp}}=4$ case when the line density increases. The resonance condition is met when the mode2 frequency shifts downwards and eventually encounters the resonance line, $\omega_{2}=2$, as $\Lambda$ approaches 0.45 . As shown in Fig. 6(c), the 1D chain configuration is completely destroyed at $\Lambda \approx 0.45$ when the emittance blows up.

$$
\text { D. } N_{\text {sp }}=3
$$

The $N_{\mathrm{sp}}=3$ case appears substantially different from the others. According to Fig. 6(d), the 1D crystal is stable only for $\Lambda \leq 0.33$. However, in this region of $\Lambda$ there is no power density at half the lattice frequency, that is, 1.5. The resonance instability observed here originates from the sum of the modes near $\omega_{1}=2.5$ and $\omega_{3}=0.5$ hitting 3 .

$$
\text { E. } N_{\text {sp }}=2
$$

Similar to the $N_{\mathrm{sp}}=4$ case, the resonance condition is met when the mode- 2 frequency shifts downwards encountering the resonance line $\omega_{2}=2$ as $\Lambda$ approaches 0.45 , as shown in Fig. 6(e). When the density is increased to this value, the stable crystalline state becomes an "excited state" [11].

$$
\text { F. } N_{\mathrm{sp}}=\mathbf{1}
$$

With $N_{\text {sp }}=1$, the $1 \mathrm{D}$ crystal becomes unstable in the region $\Lambda>0.33$. Fourier analysis indicates that the phonon frequency in mode 2 does not move much from the base tune. In contrast, the frequencies in modes 1 and 3 shift upwards, especially, $\omega_{3}$ approaches 0.5 at higher line density. Thus, we conclude that the upshift of $\omega_{3}$ causes resonance. 
(a) $N_{\mathrm{sp}}=12$
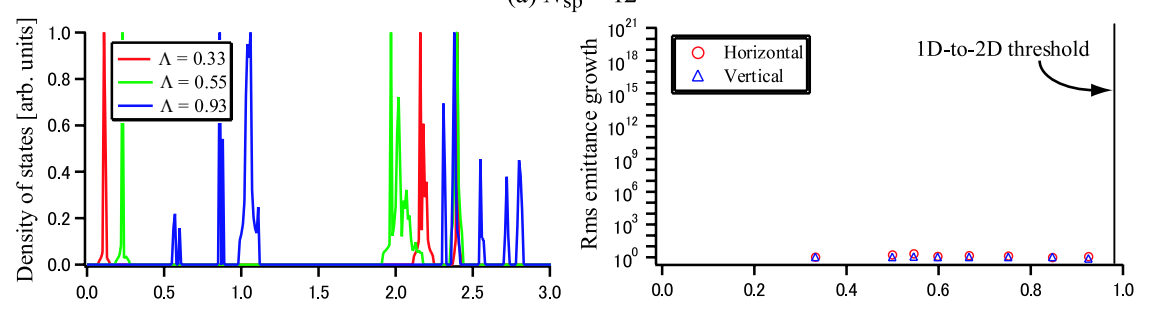

(b) $N_{\mathrm{sp}}=6$
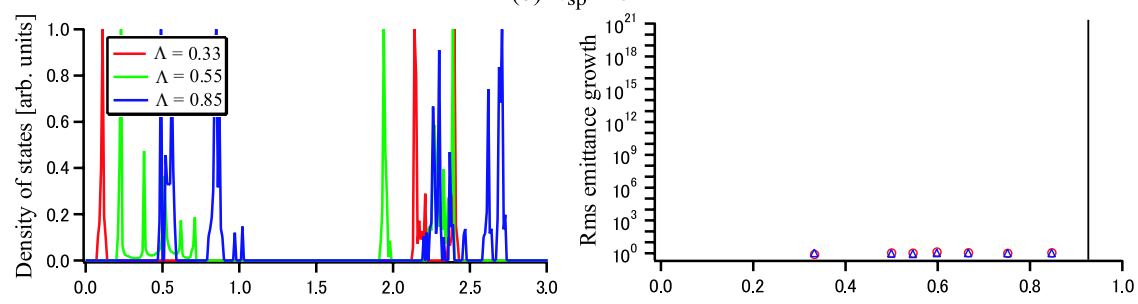

(c) $N_{\mathrm{sp}}=4$
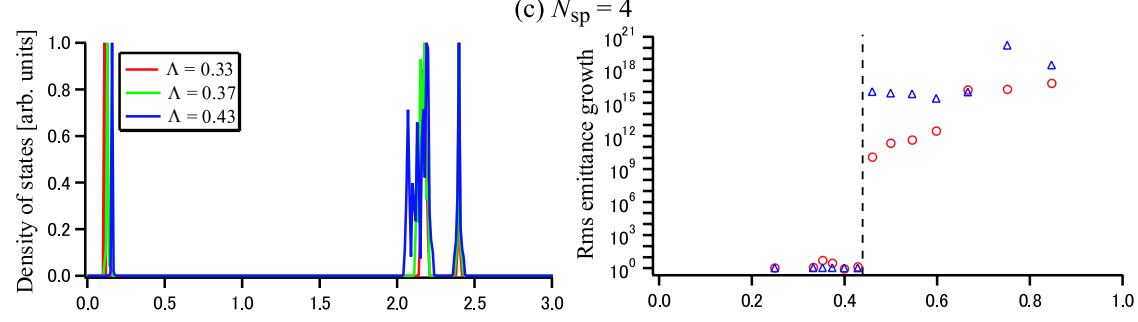

(d) $N_{\text {sp }}=3$
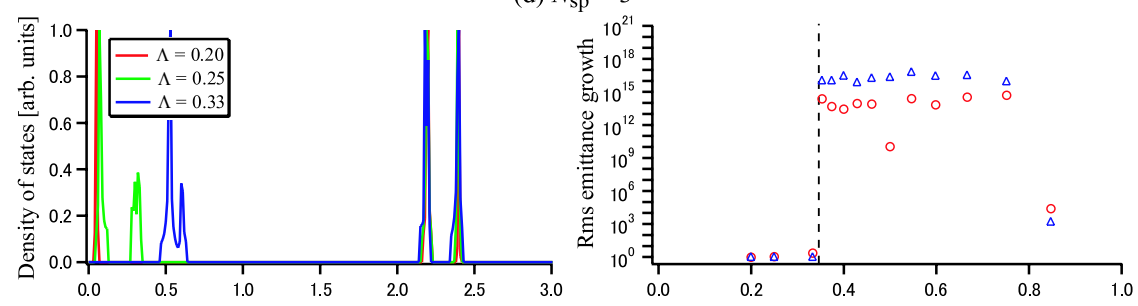

(e) $N_{\text {sp }}=2$
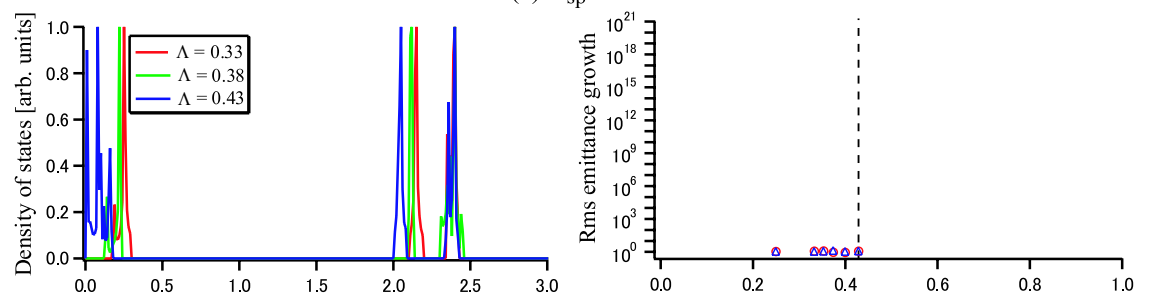

(f) $N_{\mathrm{sp}}=1$
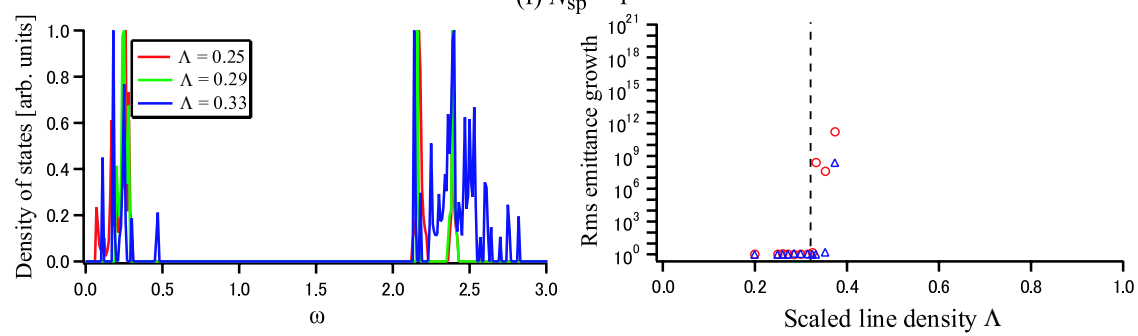

FIG. 6. (Color) Density of states and stability diagrams of 1D crystalline beams evaluated with MD simulations using MD supercells of 24 particles. FODO-based model rings with $N_{\mathrm{sp}}=12,6,4,3,2,1$ are used to verify the results in Sec. II A. In all cases, the bare betatron tunes are $\left(\nu_{x}, \nu_{y}\right)=(2.4,2.2)$. The vertical solid line in the stability diagrams indicates the threshold of $1 \mathrm{D}$-to-2D transition, while the broken line is the stability border of the $1 \mathrm{D}$ crystals. The parameter $\Lambda$ represents the scaled line density evaluated in the beam rest-frame. The actual line density in the laboratory frame is given by $\lambda=\Lambda /(\gamma \xi)$. For example, for ${ }^{24} \mathrm{Mg}^{+}$ions with a kinetic energy of $360 \mathrm{keV}(\gamma=1.000016)$ in a storage ring of $1 \mathrm{~m}$ bending radius, we obtain $\gamma \xi=12.6 \mu \mathrm{m}$. The stability of a 1D crystal at specific line density is determined by checking the rms emittance 100 turns after removing the cooling force. 
We note that the threshold density corresponding to the 1D-to-2D transition obtained with the MD simulation using the actual storage-ring lattice agrees with the analytical predictions [Eqs. (12) and (13)] using the smooth approximation within about $10 \%$.

\section{STABILITY OF MULTIDIMENSIONAL CRYSTALS}

We now study the stability of crystalline beams with nonzero transverse dimensions in the ground state. To explore the dependence of stability on the machine's latti- ces, we consider six storage rings that have different lattice functions, as displayed in Fig. 7. CRYRING [12], TARN II [13], and S-LSR [14] represent the cooler ring operating, already shut down, and under construction, respectively. Their lattice structures have sixfold symmetry. The other three, test rings I, II, and III, are the models designed solely for this simulation. Table I lists some important parameters. The horizontal and vertical tunes are set equal, i.e., $\nu_{x}=\nu_{y}\left(\equiv \nu_{0}\right)$. The simulated beams are assumed to be ${ }^{24} \mathrm{Mg}^{+}$ions traveling with a kinetic energy of $1 \mathrm{MeV}$.

Figure 8 has the stability diagrams obtained through MD simulations. To test the beam's stability, we adopt the
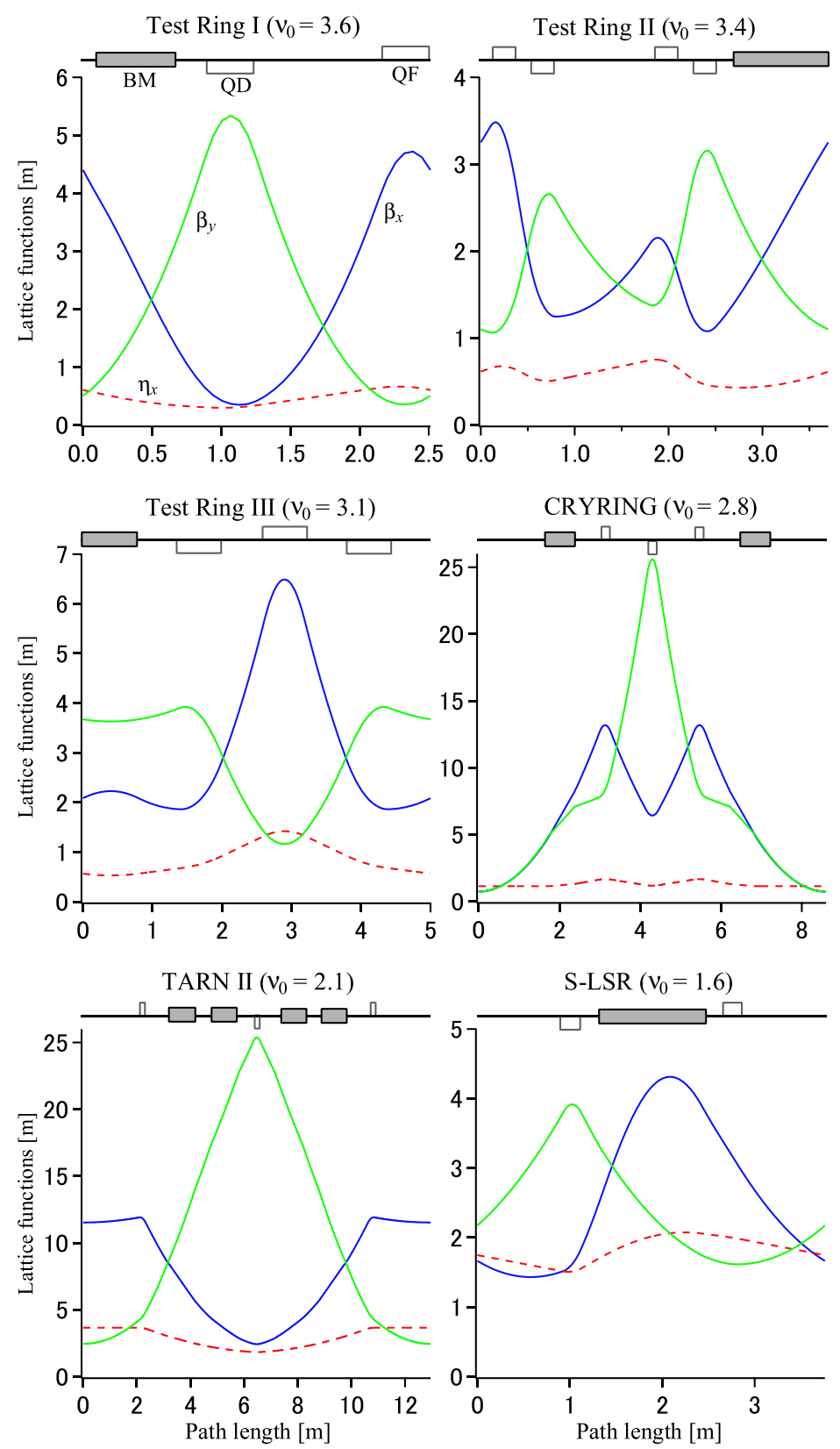

FIG. 7. (Color) Lattice functions of the test rings considered in Sec. IV. $\beta_{x(y)}$ is the horizontal (vertical) betatron function, and $\eta_{x}$ the dispersion function. All the transverse tunes are set equal $\left(\nu_{x}=\nu_{y} \equiv \nu_{0}\right)$. 
TABLE I. Main lattice parameters of six storage rings considered in Sec. IV.

\begin{tabular}{lcccccc}
\hline \hline Lattice & Ring I & Ring II & Ring III & CRYRING & TARN II & S-LSR \\
\hline Superperiodicity $N_{\mathrm{sp}}$ & 10 & 10 & 10 & 6 & 6 & 6 \\
Circumference [m] & 25.13 & 37.00 & 50.00 & 51.60 & 77.70 & 22.56 \\
Bare betatron tunes $\nu_{0}$ & $2.8 \sim 3.9$ & $3.4 \sim 3.9$ & $3.1 \sim 3.9$ & $2.0 \sim 2.8$ & $1.6 \sim 2.3$ & $1.6 \sim 2.4$ \\
\hline \hline
\end{tabular}
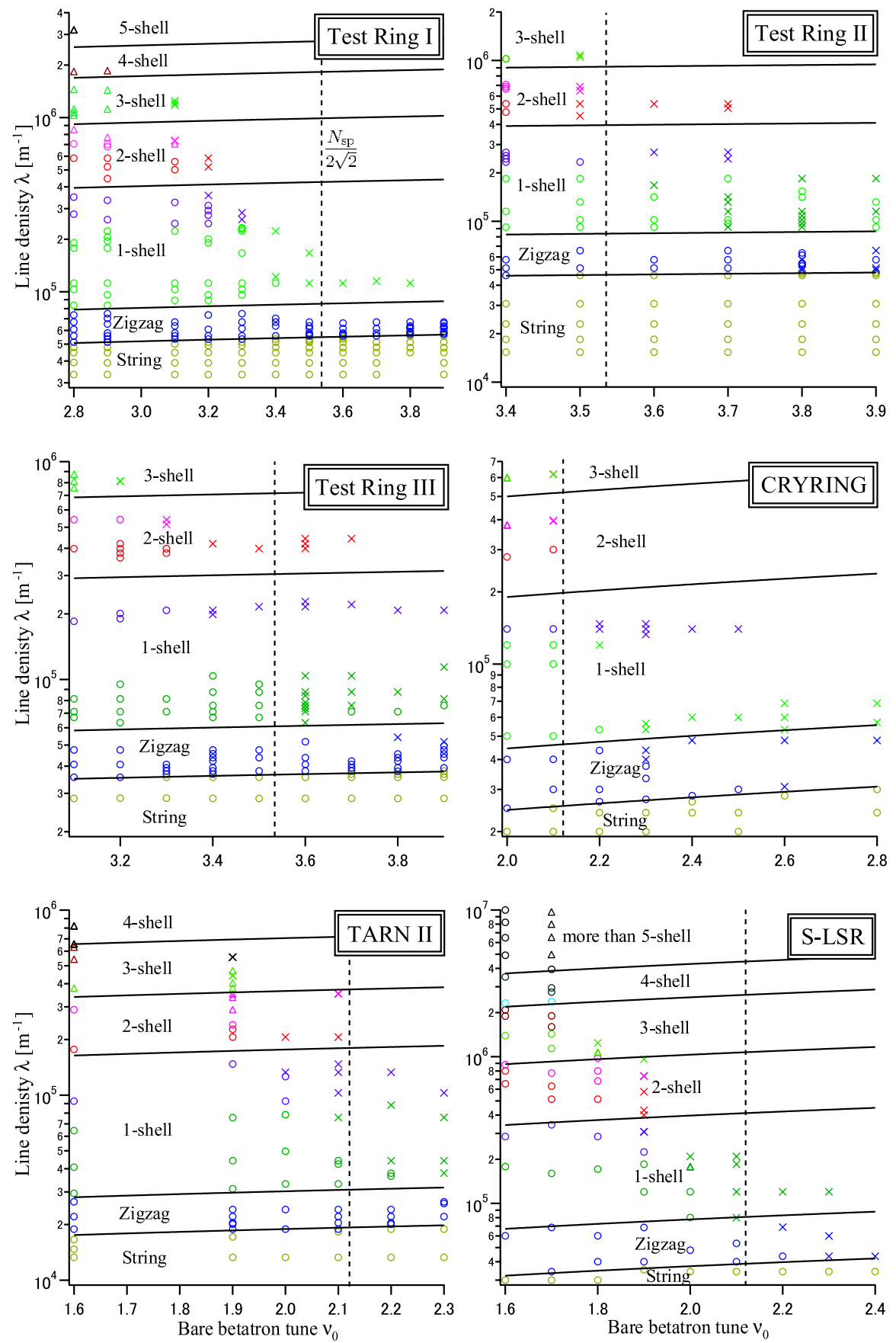

FIG. 8. (Color) Stability diagram of crystalline beams obtained by MD simulations. The "survival time" of a crystalline beam after removing dissipation is represented by either a circle $(\bigcirc)$, a triangle $(\triangle)$, or a cross $(\times)$. $\bigcirc$ stands for stable over $10^{4}$ turns, $\triangle$ for melt before $10^{4}$ turns, and $\times$ for blow-up immediately after removing the cooling force. Different colors correspond to different crystalline structures. The vertical broken line in each panel is $\nu_{0}=N_{\mathrm{sp}} / 2 \sqrt{2}$. 
following procedures: First, a strong 3D dissipative force is applied to a beam of particular line density. After a crystalline state is reached, the cooling force is removed. (If an ordered configuration cannot be established, we simply leave the corresponding operating point on the stability chart open.) When the beam is stable over $10^{4}$ turns after removing the cooling force, we mark a circle on the chart. If the "survival time" is below $10^{4}$ turns, then a triangle is placed. A cross on the chart means that the crystal at the point melted immediately after we stopped cooling. The figure indicates that $3 \mathrm{D}$ crystals generally cannot be stabilized once $\nu_{0}$ exceeds around $N_{\mathrm{sp}} / 2 \sqrt{2}$. Specifically, a multishell configuration with higher line density is less stable than a 1D one with lower line density; the stability clearly is improved by reducing the bare tunes. As expected, 1D crystals are quite stable compared to the other multidimensional configurations. Since the phonon spectrum of a 1D crystalline chain is discrete, unlike those of 3D crystals as shown in Fig. 4, the 1D crystal can remain free from resonance even in a high- $\nu_{0}$ (higher than $N_{\mathrm{sp}} / 2 \sqrt{2}$ ) region. Such features are shared by some crystals of intermediate line densities with, e.g., 2D zigzag ground-state structures.

The condition stated above, i.e. $\nu_{0}<N_{\mathrm{sp}} / 2 \sqrt{2}$, is derivable from the well-known envelope equations [15] under the smooth approximation; it is identical to the condition that avoids the excitation of linear coherent resonance at the space-charge limit. Therefore, we also investigated whether the stability of 3D crystalline states can be determined by a standard beam-dynamics theory instead of by the phonon theory. To obtain the stop bands of linear and nonlinear resonances, we numerically solved the linearized 2D Vlasov-Poisson equations employing the KapchinskyVladimirsky (KV) model as a stationary state [16]. However, the stop band distribution derived from the Vlasov analysis does not always explain the MD results in Fig. 8 (see the appendix); the Vlasov prediction contradicts the MD results in two of the six cases (Ring II and CRYRING). We speculate that the longitudinal modes neglected in the 2D Vlasov theory play a role when ordering starts to appear among the particles.

Finally, we briefly discuss the MD results based on the lattice of two European storage ring where considerable experimental effort had been devoted in 1990's to generating an ordered beam with the laser cooling technique $[17,18]$. This advanced cooling method was first successfully applied to circulating ion beams by the TSR group at the Max Planck Institute in Heidelberg [17], and later by the ASTRID group at Aarhus University in Denmark [18]. However, beam ordering has not yet been observed in either machines.

The ASTRID ring lattice is fourfold symmetric, i.e., $N_{\text {sp }}=4$, and a typical set of transverse tunes are $\nu_{x}=$ 2.6 and $\nu_{y}=1.1$. The general maintenance condition for a $3 \mathrm{D}$ crystal (14), $\sqrt{\nu_{x}^{2}+\nu_{y}^{2}}<2$, is not satisfied. Indeed, we fail to form any 3D crystalline structure even with a strong cooling force in MD simulations. At low densities, 1D string crystals and 2D zigzag crystals extending in the $y$ direction are obtained with the ideal lattice [19].

The TSR ring lattice is twofold symmetric, i.e., $N_{\mathrm{sp}}=2$, and a typical set of transverse tunes are $\nu_{x}=2.6$ and $\nu_{y}=$ 2.2. The general maintenance condition for a $3 \mathrm{D}$ crystal, $\sqrt{\nu_{x}^{2}+\nu_{y}^{2}}<1$, is again not satisfied. Not surprisingly, we again fail to form any 3D crystalline structure in MD simulations. At low densities, 1D string crystals are obtained with the ideal lattice. Beyond $1 \mathrm{D}$, the only structure attained after many attempts is an unusual zigzag structure that rotates along the longitudinal axis one revolution per lattice period [19]. Although we succeeded in forming such configurations, the line density has to be sufficiently low, and the cooling force applied is unrealistically strong [20]. We thus expect that, in reality, it is extremely difficult to reach a crystalline state in ASTRID and TSR.

\section{DISCUSSIONS AND SUMMARY}

In this paper, we focused on the maintenance condition for a crystalline beam. We derived the phonon spectrum in the smooth approximation and then compared it with that obtained by Fourier transform from MD calculations. Since the maintenance condition is primarily related to the high frequency phonon modes and the dispersion of high frequency modes are generally small, the number of particles used in such MD simulations (24 particles in a supercell in the present 1D case and several hundred in 3D multishell cases) are adequate. Test simulation shows that phonon density of states are not affected by increasing the supercell size except near zero frequency. The maintenance condition, based on the linear-resonance criteria, is that the lattice superperiodicity must not be equal to the sum of any two phonon frequencies, i.e., $N_{\text {sp }} \neq$ $\omega_{i}+\omega_{j}(i, j=1,2,3)$. We also found that the maximum phonon frequency is always below $\sqrt{\nu_{x}^{2}+\nu_{y}^{2}}$. Since the phonon spectrum of a 3D crystal is continuous, this condition states that, for crystalline beams of high line-density, half of the lattice superperiodicity must be greater than $\sqrt{\nu_{x}^{2}+\nu_{y}^{2}}$

$$
N_{\mathrm{sp}}>2 \sqrt{\nu_{x}^{2}+\nu_{y}^{2}}
$$

When the two tunes are nearly equal, this leads to the previously derived maintenance criterion $\nu_{0}<N_{\text {sp }} / 2 \sqrt{2}$ [3]. The formalism can be applied to crystal formation in traps where $\gamma=0$; a similar maintenance condition holds.

Finally, we note that we paid little attention to whether an ordinary high-temperature beam can be cooled to a crystalline state with a realistic dissipative force. Since resonance conditions of collective modes generally depend on the beam's density, the present stability criteria may have to be modified in practice. A recent simulation study 


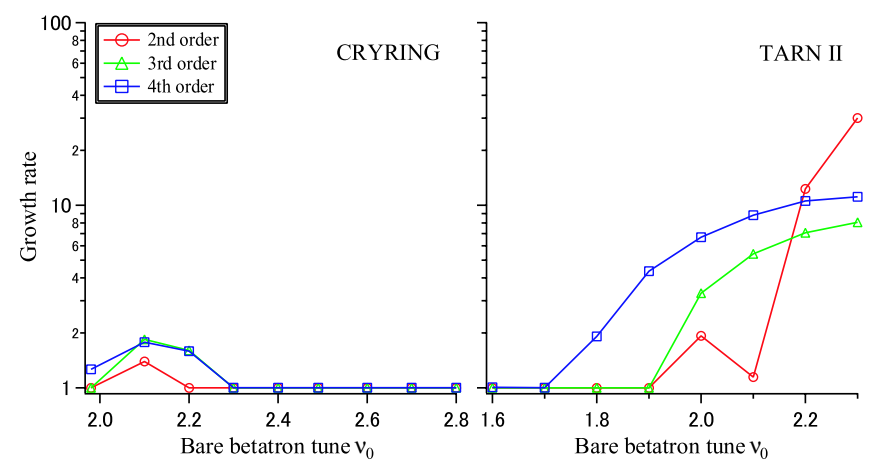

FIG. 9. (Color) Growth rates calculated by the linearized Vlasov analysis. The lattices of CRYRING and TARN II with the parameters in Table I were assumed. Resonance stop bands of three different orders are plotted as a function of $\nu_{0}$. The most dangerous resonance is that of the second order.

concluded that a complete suppression of linear resonances over the whole density region may be needed for an efficient cooling of the beam [21]. To meet this requirement, we need $\nu_{0}<N_{\mathrm{sp}} / 4$, which is more stringent than the criterion stated in Eq. (15).

We thank A. Woodhead for reading and revising the manuscript.

\section{APPENDIX: STOP BANDS FROM LINEARIZED VLASOV ANALYSIS}

At high line-density, a crystalline beam has a multishell configuration within which particles are almost uniformly distributed and no random Coulomb collisions are present. Therefore, we expected that the Vlasov analysis based on the KV model [16] might be employed to predict the transverse stability of 3D crystalline states. To check this expectation, we evaluated the growth rates of linear and nonlinear collective resonances by applying perturbation to the stationary KV beam [22]. The results of this linearized Vlasov analysis are given in Fig. 9 where resonance stop bands of three lowest orders are plotted as a function of $\nu_{0}$. Since the crystalline beam is exactly at the space-charge limit, we assumed that the effective betatron tunes are equal to zero. In the case of TARN II, the second-order resonance most dangerous to stability occurs when the bare tune exceeds about 2.0. Recalling that $N_{\mathrm{sp}}=6$ in TARN II, this observation agrees with the phonon theory's prediction, i.e., Eq. (15). However, the stop band distribution of CRYRING, which has the same superperiodicity as TARN II, cannot explain the MD results in Fig. 8. One possible reason for that could be the neglect of the longitudinal modes in the Vlasov theory. We thus conclude that the 2D Vlasov analysis has limitations in predicting the transverse stability of a crystalline beam.

\section{ACKNOWLEDGMENTS}

Work performed under the auspices of the U.S. Department of Energy.
[1] J. Wei, X.-P. Li, and A.M. Sessler, Phys. Rev. Lett. 73, 3089 (1994).

[2] X.-P. Li, A. M. Sessler, and J. Wei, in Proceedings of the European Particle Accelerator Conference, edited by V.P. Suller and Ch. Petit-Jean Genaz (World Scientific, Singapore, 1994), p. 1379.

[3] J. Wei, H. Okamoto, and A. M. Sessler, Phys. Rev. Lett. 80, 2606 (1998).

[4] F. Diedrich, E. Peik, J. M. Chen, W. Quint, and H. Walther, Phys. Rev. Lett. 59, 2931 (1987).

[5] D. J. Wineland, J.C. Bergquist, W.M. Itano, J.J. Bollinger, and C. H. Manney, Phys. Rev. Lett. 59, 2935 (1987).

[6] M. Drewsen, C. Brodersen, L. Hornekaer, J. S. Hangst, and J. P. Schiffer, Phys. Rev. Lett. 81, 2878 (1998).

[7] H. Okamoto and J. Wei, Phys. Rev. E 58, 3817 (1998).

[8] Y. Yuri and H. Okamoto, Phys. Rev. ST Accel. Beams 8, 114201 (2005).

[9] U. Schramm, M. Bussmann, and D. Habs, Nucl. Instrum. Methods Phys. Res., Sect. A 532, 348 (2004); see, also, T. Schätz, U. Schramm, and D. Habs, Nature (London) 412, 717 (2001).

[10] J. Wei, X.-P. Li, and A. M. Sessler, Brookhaven National Laboratory Report No. BNL-52381, 1993.

[11] We observe with the MD simulations that when the density of the crystal meets the phonon resonance condition, the crystal becomes an unusual 2D zigzag structure where the positions of the two nearby particles in the transverse direction oscillate and switch with each other within the lattice period. This so-called "excited state" is to be studied further.

[12] K. Abrahamsson et al., Nucl. Instrum. Methods Phys. Res., Sect. B 79, 269 (1993).

[13] T. Katayama et al., in Proceedings of the 2nd European Particle Accelerator Conference, edited by P. Martin and P. Mandrillon (Editions Frontières, Gif-sur-Yvette, 1990), p. 577.

[14] A. Noda, Nucl. Instrum. Methods Phys. Res., Sect. A 532, 150 (2004).

[15] F. J. Sacherer, IEEE Trans. Nucl. Sci. 18, 1105 (1971).

[16] I. M. Kapchinskij and V. V. Vladimirskij, in Proceedings of the Internatinal Conference on High Energy Accelerators (CERN, Geneva, 1959), p. 274.

[17] S. Schröder et al., Phys. Rev. Lett. 64, 2901 (1990).

[18] J. S. Hangst, M. Kristensen, J. S. Nielsen, O. Poulsen, J.P. Schiffer, and P. Shi, Phys. Rev. Lett. 67, 1238 (1991).

[19] J. Wei, X.-P. Li, and A. M. Sessler, in Proceedings of the 1995 Particle Accelerator Conference and International Conference on High-Energy Accelerators (IEEE, Dallas, Texas, 1995), p. 2946.

[20] In these MD simulations, we used so-called "periodic cooling" to reach the ground-state structure, i.e., imposing the periodic conditions on each coordinate every lattice period and, at the same time, correcting the momentum of each particle to compensate the drift in the longitudinal direction [1].

[21] K. Okabe and H. Okamoto, Jpn. J. Appl. Phys. 42, 4584 (2003).

[22] I. Hofmann, L. Laslett, L. Smith, and I. Haber, Part. Accel. 13, 145 (1983). 